\title{
High-throughput DNA methylation analysis in anorexia nervosa confirms TNXB hypermethylation
}

\author{
Miriam Kesselmeier ${ }^{\mathrm{a}}$, Carolin Pütter ${ }^{\mathrm{b}}$, Anna-Lena Volckmar ${ }^{\mathrm{c}}$, Hansjörg Baurecht ${ }^{\mathrm{d}}$, Harald Grallert ${ }^{\mathrm{e}, \mathrm{f}}$, \\ Thomas Illig ${ }^{\mathrm{e}, \mathrm{g}, \mathrm{h}}$, Khadeeja Ismail', Miina Ollikainen', Yasmina Silén', Anna Keski-Rahkonen', \\ Cynthia M. Bulik, ${ }^{\mathrm{j}, \mathrm{k}}$, David A. Collier, ${ }^{\mathrm{l}, \mathrm{m}}$, Eleftheria Zeggini ${ }^{\mathrm{n}}$, Johannes Hebebrand ${ }^{\mathrm{c}}$, André Scherag ${ }^{\mathrm{a} *}$, \\ Anke Hinney ${ }^{\mathrm{C}}$ and $\mathrm{GCAN}^{\dagger}$ and WTCCC3 $^{\dagger}$
}

\begin{abstract}
${ }^{a}$ Clinical Epidemiology, Integrated Research and Treatment Center, Center for Sepsis Control and Care (CSCC), Jena University Hospital, Jena, Germany; Institute for Medical Informatics, Biometry and Epidemiology, University of Duisburg-Essen, Essen, Germany;

${ }^{\circ}$ Department of Child and Adolescent Psychiatry, Psychosomatics and Psychotherapy, University Hospital Essen, University of DuisburgEssen, Essen, Germany; 'Department of Dermatólogy, Allergology, and Venereology,' University Hospital Schleswig-Holstein, Campus

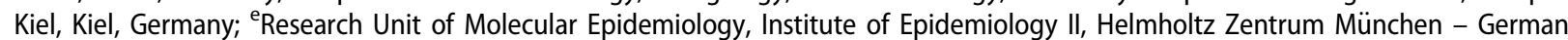
Research Center for Environmental Health, Neuherberg, Germany; ${ }^{f}$ German Center for Diabetes Research, Neuherberg, Germany;

${ }^{g}$ Hannover Unified Biobank, Hannover Medical School, Hannover, Germany; hnstitute of Human Genetics, Hannover Medical School, Hannover, Germany; 'Department of Public Health, University of 'Helsinki, Helsinki, Finland; 'Department of Psychiatry, University of

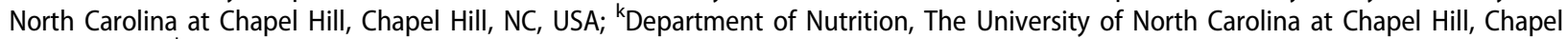
Hill, NC, USA; 'Social, Genetic and Developmental Psychiatry Centre, Institute of Psychiatry, King's College London, London, UK; ${ }^{m}$ Eli Lilly and Company, Erl Wood Manor, Windlesham, UK; ' Wellcome Trust Sanger Institute, Wellcome Trust Genome Campus, Hinxton, Cambridge, UK
\end{abstract}

\begin{abstract}
Objectives: Patients with anorexia nervosa (AN) are ideally suited to identify differentially methylated genes in response to starvation.

Methods: We examined high-throughput DNA methylation derived from whole blood of 47 females with AN, 47 lean females without AN and 100 population-based females to compare AN with both controls. To account for different cell type compositions, we applied two referencefree methods (FastLMM-EWASher, RefFreeEWAS) and searched for consensus CpG sites identified by both methods. We used a validation sample of five monozygotic AN-discordant twin pairs.

Results: Fifty-one consensus sites were identified in AN vs. lean and 81 in AN vs. populationbased comparisons. These sites have not been reported in AN methylation analyses, but for the latter comparison 54/81 sites showed directionally consistent differential methylation effects in the AN-discordant twins. For a single nucleotide polymorphism rs923768 in CSGALNACT1 a nearby site was nominally associated with AN. At the gene level, we confirmed hypermethylated sites at $T N X B$. We found support for a locus at $N R 1 H 3$ in the AN vs. lean control comparison, but the methylation direction was opposite to the one previously reported.

Conclusions: We confirm genes like TNXB previously described to comprise differentially methylated sites, and highlight further sites that might be specifically involved in AN starvation processes.
\end{abstract}

\section{ARTICLE HISTORY}

Received 6 November 2015

Revised 12 April 2016

Accepted 9 May 2016

\section{KEYWORDS}

Anorexia nervosa; DNA methylation; eating disorder; epigenome-wide association study; starvation

\section{Introduction}

Anorexia nervosa (AN) is a pernicious condition characterized by restriction of energy intake and extremely low body weight. Epigenetic alterations are assumed to play a key role in moderating or mediating the impact of environmental/lifestyle exposures on gene function and can also be influenced by genetic variations (Haggarty 2015). Starvation is a key clinical feature of patients with $\mathrm{AN}$ and a strong environmental exposure can be expected to trigger epigenetic alterations (Heijmans et al. 2008). DNA methylation is one frequently investigated epigenetic mechanism, which is known to change over time (Fraga et al. 2005) and can also be influenced by the metabolome (Keating \& ElOsta 2015). Previous investigations of DNA methylation in AN mainly focused either on the (promoter) methylation of specific candidate genes (Campbell et al. 2011; Pjetri et al. 2012) or on markers for a 
Table 1. Participant characteristics of the high-throughput DNA methylation analysis and the validation datasets.

\begin{tabular}{|c|c|c|c|c|c|}
\hline & \multirow[b]{2}{*}{ AN cases $(n=47)$} & \multirow[b]{2}{*}{ LEAN controls $(n=47)$} & \multirow[b]{2}{*}{ POP controls $(n=100)$} & \multicolumn{2}{|c|}{ Replication data ( $n=5 \mathrm{MZ}$ pairs) } \\
\hline & & & & AN co-twin & Healthy co-twin \\
\hline Sex (females); n (\%) & $47(100)$ & $47(100)$ & $100(100)$ & \multicolumn{2}{|c|}{5 pairs $(100)$} \\
\hline \multicolumn{6}{|l|}{ AN type; $n(\%):$} \\
\hline Restricting & $36(76.6)$ & & & $4(80)$ & \\
\hline Binge-eating/purging & $8(17.0)$ & & & $1(20)$ & \\
\hline Missing & $3(6.4)$ & & & $0(0)$ & \\
\hline Age (years); median (Q1, Q3) & $16(14,17)$ & $22(21,23)$ & $60(54,69)$ & \multicolumn{2}{|c|}{$22(22,23)$} \\
\hline Restricting type AN & $15(14,17)$ & & & $22(22,22)$ & \\
\hline Binge-eating/purging type AN & $16(15,17)$ & & & $28(-)$ & \\
\hline BMI $\left(\mathrm{kg} / \mathrm{m}^{2}\right)$; median $(\mathrm{Q} 1, \mathrm{Q} 3)$ & $13.7(12.0,14.6)$ & $17.3(16.9,17.7)$ & $26.6(24.3,32.1)$ & $22.0(21.5,23.3)$ & $20.7(20.2,24.5)$ \\
\hline Restricting AN type & $13.7(13.0,14.5)$ & & & $21.8(20.7,22.3)$ & \\
\hline Binge-eating/purging AN type & $12.7(12.1,14.0)$ & & & $29.8^{\mathrm{a}}(-)$ & \\
\hline
\end{tabular}

global methylation pattern (Saffrey et al. 2014). Recently, Booij et al. (2015) described a high-throughput DNA methylation analysis (450K Illumina bead arrays from lymphocytes) in 29 female patients with acute $\mathrm{AN}$ and 15 normal-weight female controls and reported 14 hypermethylated AN CpG sites at 11 genes (PRDM16, HDAC4, TNXB, FTSJD2, PXDNL, DLGAP2, FAM83A, NR1H3, DDX10, ARHGAP1, PIWIL1). Note that such high-throughput DNA methylation analyses rely on the pre-selected content of methylation sites (for details on technical properties of the 450K array: e.g., Dedeurwaerder et al. 2011).

Here, we analyse high-throughput DNA methylation data derived from whole blood for differences between AN patients and two control groups: lean (body mass index $[\mathrm{BMI}] \leq 15$ th age- and sex-specific percentile) and population-based female controls. We included two control groups as they highlight different aspects of potential differential DNA methylation: the comparison of AN patients vs. lean females without AN will allow for assessing starvation as a key clinical feature of AN, while the comparison of AN patients vs. population-based female controls (without any weight restrictions) will allow for more broadly analysing the general aspects of AN. Lastly we compared methylation pattern in both control groups; these should not be different at the sites specific for AN.

To correct for known cell type distribution effects under starvation (Fukudo et al. 1993; Saito et al. 1999; Castro et al. 2004; Sabel et al. 2013; Bühren et al. 2014), we applied two recently proposed referencefree methods (Houseman et al. 2014; Zou et al. 2014) and investigated their overlapping results. We aimed to identify patterns of differential DNA methylation contrasting AN patients and controls. We discuss our findings on the background of a systematic literature search and data of the most recent and largest genome-wide association meta-analysis (GWAMA; Boraska et al. 2014) on 2,907 AN cases and 14,860 controls conducted by the Genetic Consortium for Anorexia Nervosa (GCAN) as part of the Wellcome Trust Case Control Consortium 3 (WTCCC3) to identify previously reported findings or potential overlapping epigenetic and genetic alterations associated with acute AN.

\section{Materials and methods}

\section{Study subjects}

We analysed 47 females with AN, 47 lean females (BMI $\leq 15$ th age- and sex-specific percentile) without $A N$, and 100 population-based females (POP; Table I). Females with AN prior to weight restoration were recruited and diagnosed (DSM-IV criteria fulfilled) at the Departments of Child and Adolescent Psychiatry of the Philipps-University of Marburg and of the University of Duisburg-Essen. All AN patients were interviewed with either the Composite International Diagnostic Interview (CIDI) or the Diagnostic Interview for Genetic Studies and the Eating Disorder Family History Interview (for details see Hinney et al. 1997). Lean females (BMI $\leq 15$ th percentile) without AN were recruited among the students of the Philipps-University of Marburg. They were reimbursed for their voluntary participation, had to have no somatic disorders and had to consume $\leq 10$ cigarettes per day. Furthermore, the lean females were screened as follows: (1) life-time occurrence of $\mathrm{AN}$ and bulimia nervosa by the eating disorder section of the CIDI, (2) the German version of the Three-Factor Eating Questionnaire to ensure that only non-restrained eaters defined as individuals who scored five or less on the cognitive restraint factor were included, and (3) for their weight history by a semi-structured interview. For details we again refer to Hinney et al. (1997). Lean females that either fulfilled (1) or (2) or who reported that they had a higher 
weight than same-aged individuals at ages 10,15 and/ or 18 were excluded. The 100 POP were a random sample from a dataset of 925 females of the population-based Cooperative Health Research in the Region of Augsburg (KORA) Survey F4 (Dick et al. 2014). Details on this part of the KORA cohort with a focus on high-throughput methylation profiling are provided in Dick et al. (2014). Control group participants were not specifically assessed for eating disorders. In the following sections, the group of females with $A N$ is abbreviated by AN, the group of lean females without AN by LEAN and the population-based female controls selected from the KORA survey by POP. All studies were approved by the relevant institutional ethics committees, and all women provided written informed consent; the study was conducted in accordance to the Declaration of Helsinki.

\section{High-throughput methylation data generation and pre-processing}

High-throughput methylation profiling of whole blood of all samples was performed using Illumina HumanMethylation450 bead arrays at the German Center for Diabetes Research, Neuherberg (for details see Dick et al. 2014). The raw data were transformed into beta values using Illumina's Genome Studio methylation module according to the manufacturer's recommendation. We ran the pipeline of Touleimat and Tost (2012) for quality control (correction for batch effects, deletion of non-high-confidence probes), probe filtering (removing probes containing or extending on single nucleotide polymorphisms (SNPs) with minor allele frequencies of at least $5 \%$ in the Caucasian population to reduce the influence of genetic variations on methylation level variation), signal correction (colourbias adjustment, background level correction based on negative control probes) and subset-based quantile normalisation (Infl/Infll shift correction, betweensample normalisation).

\section{Cell type composition and high-throughput methylation association analyses}

Methylation differences were investigated for two between-group comparisons: (1) AN vs. LEAN and (2) AN vs. POP. First, we used the high-throughput methylation data to estimate the individual cell type compositions applying the algorithm by Houseman et al. (2012) and the reference dataset provided by Reinius et al. (2012). Secondly and based on these results, the cell type admixture had to be taken into account for the high-throughput methylation association analyses.
We applied two recently proposed reference-free methods to address the cell type admixture bias (FastLMM-EWASher, Zou et al. 2014; RefFreeEWAS, Houseman et al. 2014). We followed the recommendations by the authors of FastLMM-EWASher (Zou et al. 2014) and considered only those CpG sites with an average beta value across all samples between 0.1 and 0.9 . We required that the genomic control factor $\lambda$ had to be below 1.2 and a maximum of 10 principal components were allowed to be fitted. We decided to adjust for no further covariates even though the POP were for example considerably older than both the AN patients and the lean females. This decision was based on the observation that an inclusion of "age" would lead to an almost complete separation of the casecontrol groups resulting in unstable estimates. For both methods we applied an exploratory two-sided significance level $\alpha$ of 0.01 to detect CpG sites that are potentially differentially methylated between two groups. We then compared the results of FastLMMEWASher and RefFreeEWAS for the two between-group comparisons at each CpG site. We call CpG sites identified by both reference-free methods "consensus CpG sites" and focused on them for robustness reasons. Supplementary Figure S1 (available online) is a flowchart of our procedure. As sensitivity analyses, these consensus CPG sites were assessed for quantitative age and BMI effects, potential menstruation effects and effects related to smoking (Supplementary Tables S8-S11 available online). Conversely, we screened the literature on these and related phenotypes and their overlap to our consensus CpG sites (Supplementary Table S12 available online). As another sensitivity analysis, we also performed a third between-group comparison (LEAN vs. POP) using the same approach as for the other two primary comparisons.

\section{Comparison to the literature and to the $A N$ GWAMA}

We performed a systematic literature search and assessed the most recent and largest GWAMA for AN (Boraska et al. 2014).

We searched PubMed and the ISI Web of Knowledge at 2015/05/18 for articles dealing with AN or weight-loss intervention in relation to DNA methylation. The search term was "((anorexia nervosa) OR (weight-loss intervention) OR (underweight) OR (eating disorder)) AND (DNA methylation)". Articles were excluded if they: (1) exclusively dealt with animal models or (2) dealt with methylation globally (i.e., no genes were specified). Articles, which were automatically identified by the databases, were manually re-checked 


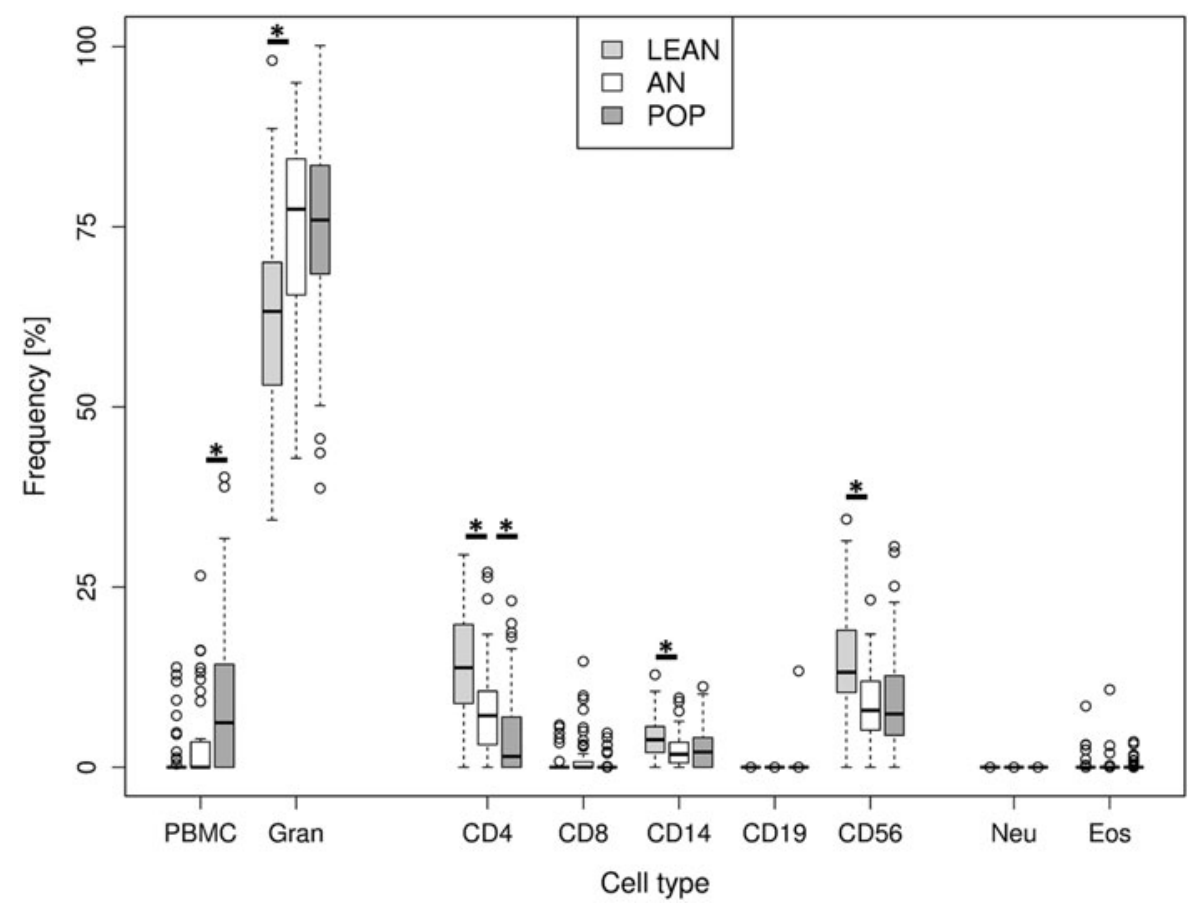

Figure 1. Boxplots of the estimated cell type distributions (between-group comparisons with $P$ values $\leq 0.01$ are indicated by asterisk; for details see Table S1).

for fulfilling the search criteria. Review articles were screened for further original research articles. From the included articles we extracted all candidate genes for which some evidence for DNA methylation was reported by the authors and reported the results for the corresponding $\mathrm{CpG}$ sites in our two between-group comparisons.

Finally, we compared the results of our consensus CPG sites with the genomic variation reported in the GCAN/WTCCC3 GWAMA by Boraska et al. (2014). More precisely, we worked with the effects of SNP associations expressed as estimated odds ratio, the corresponding nominal $P$ value under a log-additive genetic model and the SNP-wise heterogeneity statistic $\left(I^{2}\right.$ in \%) from the GCAN/WTCCC3 GWAMA. To map these statistics to our high-throughput methylation association analyses, we normalised both datasets to the same genetic map (NCBI36, hg18) and reported the results for the best SNP (the SNP with the smallest $P$ value within a maximal distance of $1 \mathrm{Mb}$ to the consensus (PG site).

\section{Validation in monozygotic twins}

We used monozygotic (MZ) twin pairs discordant for $\mathrm{AN}$ as a validation set. These twin pairs were selected from two longitudinal population-based Finnish twin cohorts, FinnTwin12 and FinnTwin16, each consisting of five consecutive birth cohorts of Finnish twins
(Kaprio 2013). Among 5,417 families we were able to identify $36 \mathrm{MZ}$ twin pairs discordant for AN (cases fulfilling the DSM5 criteria for AN). However, only five pairs were AN-discordant at the time of blood draw for the methylation analysis (Table 1). Within-pair DNA methylation differences were computed for the consensus $\mathrm{CpG}$ sites using paired moderated t tests implemented in the $\mathrm{R}$ package limma.

\section{Results}

\section{Cell type composition and high-throughput methylation association analyses}

Figure 1 displays the cell type compositions by comparison group as derived from the DNA methylation data (for details see also Reinius et al. 2012). AN and LEAN females showed group differences in the global distribution of granulocytes (Gran; median frequency: 77 vs. $63 \%$ ) and specifically in $\mathrm{CD}^{+}{ }^{+} \mathrm{T}$ cells (7 vs. $14 \%$ ), $\mathrm{CD}_{14}^{+}$monocytes (2 vs. $4 \%$ ) and $\mathrm{CD}^{+} 6^{+}$NK cells ( 8 vs. $13 \%)$. AN and POP groups differed globally in peripheral blood mononuclear cell (PBMC; 0 vs. $6 \%$ ) and specifically $\mathrm{CD}^{+} \mathrm{T}$ cell (7 vs. $2 \%$ ) distributions (Figure 1 ; detailed results in Table S1).

Next, we addressed the cell type admixture effects using two reference-free methods for the highthroughput methylation association analyses. The QQplots indicated a residual inflation of small $P$ values for RefFreeEWAS (Figure S2; Manhattan plots in Figure S3 
available online). Consequently, we identified 26,769 differentially methylated CpG sites for AN vs. LEAN according to RefFreeEWAS $(P \leq 0.01)$ but only 1,059 CpG sites for FastLMM-EWASher $(P \leq 0.01)$. For AN vs. $\mathrm{POP}$, these numbers were 11,395 and 2,607 , respectively. Fifty-one differentially methylated CpG sites were identified as consensus CPG sites by both referencefree methods for AN vs. LEAN, and 81 consensus CpG sites for AN vs. POP (Tables 2 and 3). In both cases, the number of consensus $\mathrm{CpG}$ sites significantly exceeded that expected by chance $(P<0.001)$. There were no consensus CpG sites that were identified in both group comparisons (Tables S2 and S3, available online, contrast the consensus CpG sites from the first in the second comparison and vice versa). Moreover, the consensus $\mathrm{CpG}$ sites were not previously reported in the literature on age, BMl, menstruation or smoking effects (Supplementary Table S12 available online) and our sensitivity analyses also did not strongly support such associations (except for cg25361850 (ZNF577) all $P \geq 0.05$ after table-wise multiple testing adjustment (for 132 consensus CpG sites); Supplementary Tables S8-S11 available online). Furthermore, only one consensus CpG site from AN vs. POP (cg13826718 on chromosome 13 at 36919368 without an annotated gene; both $P \leq 0.01$ ) and none from AN vs. LEAN showed a consensus association signal for LEAN vs. POP (Supplementary Tables S13 and S14, Supplementary Figures S5 and S6 available online).

\section{Comparison to the literature and to the $A N$ GWAMA}

In the systematic literature review, we identified 66 articles of which 24 met our inclusion criteria (see Figure S4, Tables S4 and S5 available online); 24 articles reported on 66 (DNA methylation) candidate genes that are summarized in Table S5 (available online). For HDAC4, NR1H3, PRDM16, TNXB, WT1 and ZNF783 we found CpG sites with suggestive evidence for a group comparison association (at $P \leq 0.01$ ) irrespective of the analysis method (bold genes in Table S5 available online). Interestingly, four of the six genes (HDAC4, NR1H3, PRDM16, TNXB) were derived from the study of Booij et al. (2015), which had a similar casecontrol study design also pertaining to patients with AN and controls. When comparing the unadjusted directions of effects, we observed that the TNXB CpG sites were hypermethylated in our AN patients compared to both control groups, which was similar to the results reported by Booij et al. (2015).

The comparison of our consensus CpG sites with the GCAN GWAMA by Boraska et al. (2014)
(Tables 2 and 3) revealed one SNP (rs923768) in the chondroitin sulphate $\mathrm{N}$-acetylgalactosaminyltransferase 1 gene (CSGALNACT1) with suggestive evidence for an association with AN susceptibility $\left(P=1.31 \times 10^{-6}\right)$. The corresponding $\mathrm{CpG}$ site was differentially methylated between the AN and POP samples. Apart from this signal, we detected no association signal meeting the threshold of suggestive evidence for an association $\left(P \leq 1 \times 10^{-5}\right)$.

\section{Validation in MZ twins}

A total of $54 / 81$ consensus CPG sites from the comparison of AN vs. POP showed mean methylation differences of the same direction in the AN-discordant $M Z$ twin sample. This indicates greater than expected overlap in the direction of methylation differences between both of the samples (AN vs. POP, and AN-discordant $\mathrm{MZ}$ twin pairs; exact binomial test: $P_{\text {sign-test }}=$ $1.69 \times 10^{-3}$ ). Of the 51 consensus $\mathrm{CpG}$ sites from the comparison AN vs. LEAN, 26 showed a consistent direction of methylation differences in the discordant $M Z$ twin sample (exact binomial test: $P_{\text {sign-test }}=1.0$ ). At the individual CpG site level, none of the tested CpG sites were differentially methylated within the twin pairs ( $P>0.01$, Supplement Tables S6 and S7).

\section{Discussion}

Epigenetic marks can be altered by both genetic and environmental factors. For example, altered epigenetic profiles associated with BMI variability have already been described (e.g., Dick et al. 2014). We hypothesized that the epigenomic pattern in weight regulation can optimally be analysed by comparing starved individuals and lean or population-based controls. As starvation is a key feature of AN, we analysed highthroughput DNA methylation data derived from whole blood for differences between female AN patients prior to weight restoration and lean or population-based female controls. Initially, we estimated that the cell type distributions derived from DNA methylation data differed between the groups. However, there was also a large overlap of the group distributions and no clear group separation in accordance with data reported previously, which did not rely on DNA methylation (Fukudo et al. 1993; Saito et al. 1999; Castro et al. 2004; Sabel et al. 2013; Bühren et al. 2014). To address the resulting cell type admixture bias in our highthroughput DNA methylation analyses, we applied two recently published reference-free methods and focused on the $\mathrm{CpG}$ sites detected by both methods. There were 51 differentially methylated consensus CpG sites 


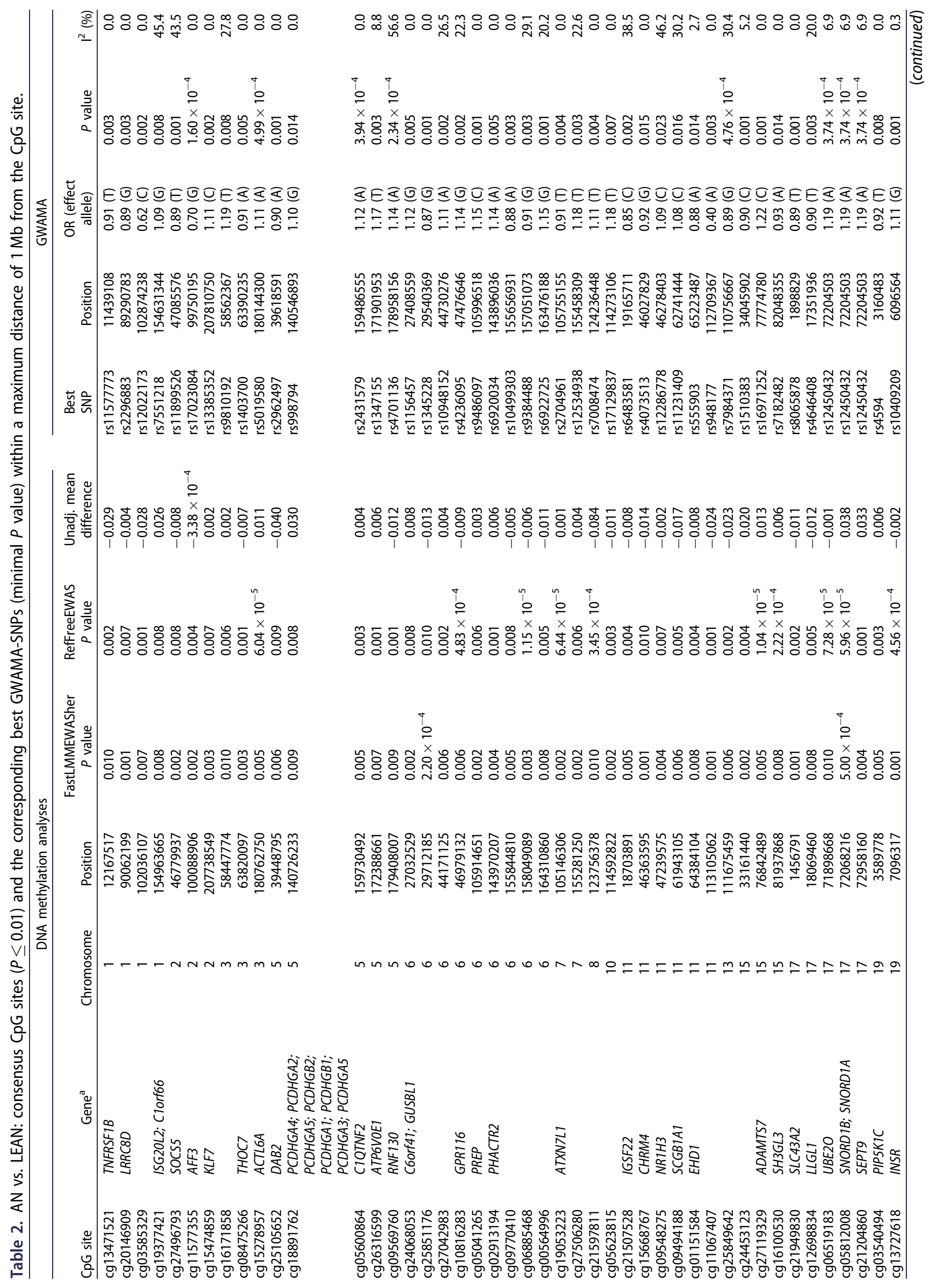




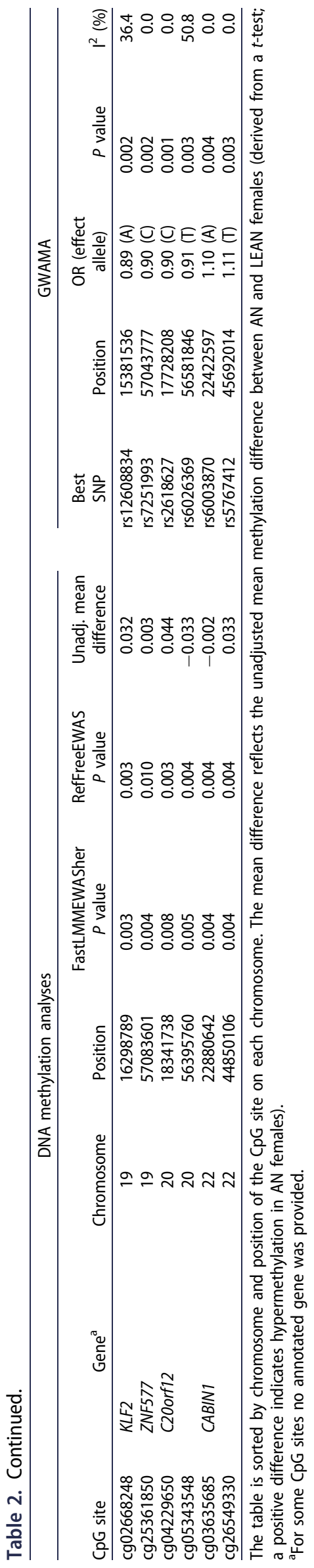

for the comparison of females with $A N$ vs. lean controls and 81 consensus CpG sites for the contrast of AN patients vs. population-based controls. None of the consensus CPG sites were detected as differentially methylated in both group comparisons and none of the individual consensus CpG sites could be validated in the AN-discordant $\mathrm{MZ}$ twin pairs. However, we observed that 54 (out of 81 ) consensus CpG sites associated with AN in the AN vs. POP comparison showed the same direction of association with AN in the validation sample.

Interestingly, moving from $\mathrm{CpG}$ sites to genes, we confirmed one previously described AN candidate gene locus. Similar to Booij et al. (2015), we detected that multiple CpG sites at the TNXB gene were hypermethylated in our AN patients compared to both our control groups. The TNXB (Tenascin XB gene) encodes an extracellular matrix glycoprotein with anti-adhesive effects (Bristow et al. 1993). Absence of the protein in humans has been associated with the Ehlers-Danlos syndrome, a connective tissue disorder (Chen et al. 2009). Localization of the gene on chromosome 6 is within the major histocompatibility complex class III region (Weissensteiner \& Lanchbury 1997). In a study based on a small sample size, gene expression levels of TNXB have been proposed as a potential diagnostic tumour marker to discriminate malignant mesothelioma from metastatic carcinoma in effusions (Yuan et al. 2009). There are hints for association of SNPs in the TNXB region with age-related macular degeneration; however, the result was not genome-wide significant (Cipriani et al 2012). It was recently shown that mutations in TNXB can cause hereditary primary vesicoureteral reflux, which is the most common congenital kidney and urinary tract anomaly (Gbadegesin et al. 2013). However, an effect on body weight regulation, starvation or AN has not yet been described.

Another interesting finding pertains to a differentially methylated $\mathrm{CpG}$ site that was recently described for AN (Booij et al. 2015). The methylation site is located at the NR1H3 (Nuclear Receptor Subfamily 1, Group $H$, Member 3 gene) gene locus. Booij et al. (2015) detected two CpG sites at the chromosomal locus; one of which is identical to the $\mathrm{CpG}$ site identified here (cg09548275). However, the direction of the effect (hyper- vs. hypomethylation) is opposite to the previous study. NR1H3 is a key regulator of macrophage function and controls transcriptional programs involved in lipid homeostasis and inflammation (Rébé et al. 2012). For $\mathrm{CD}_{14}{ }^{+}$cells (cellular marker for macrophages) our estimated cell type distributions (Figure 1) revealed a nominal difference between AN patients and lean controls (nominal $P<0.01$ ), but not for AN vs. 


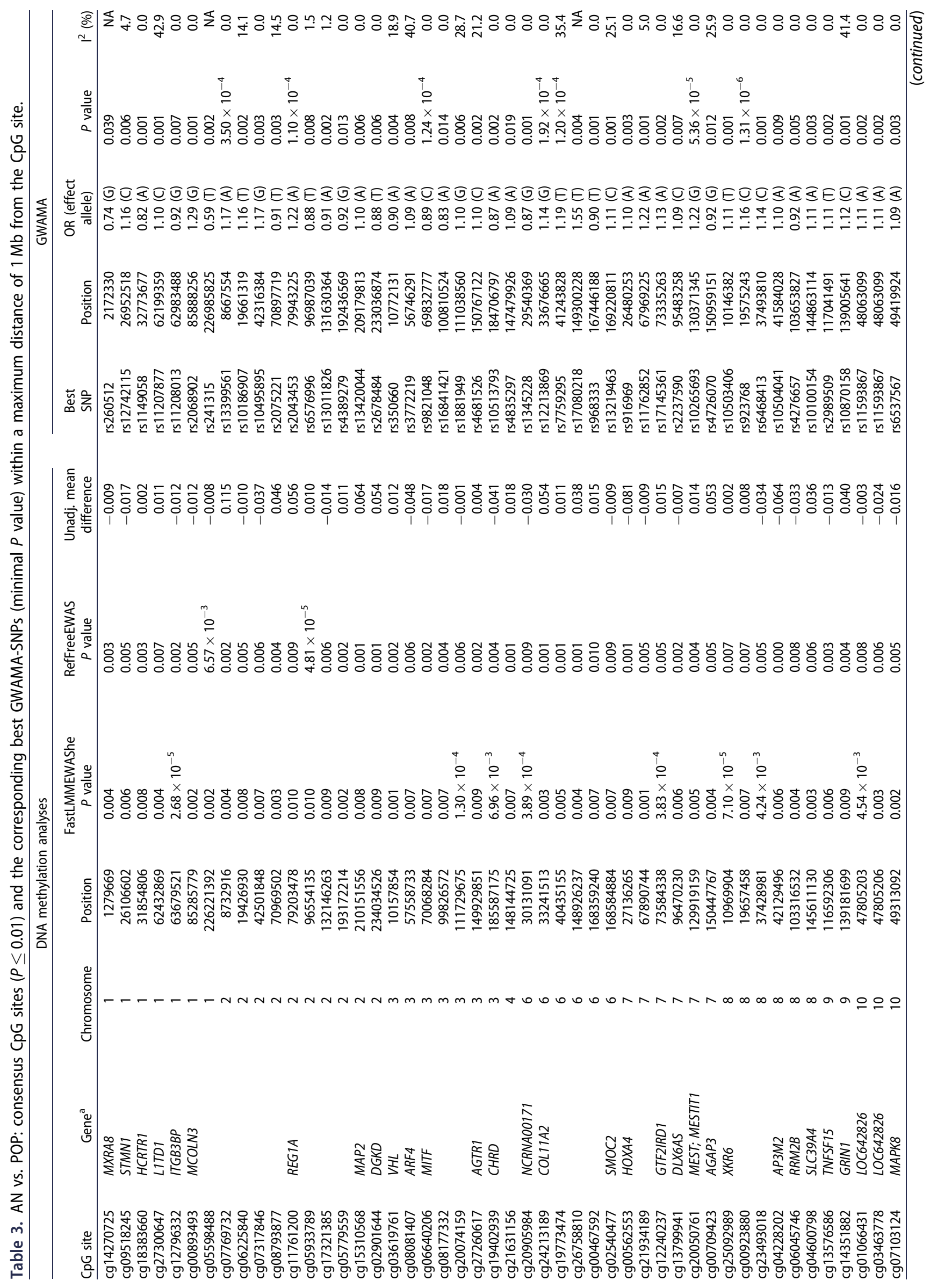




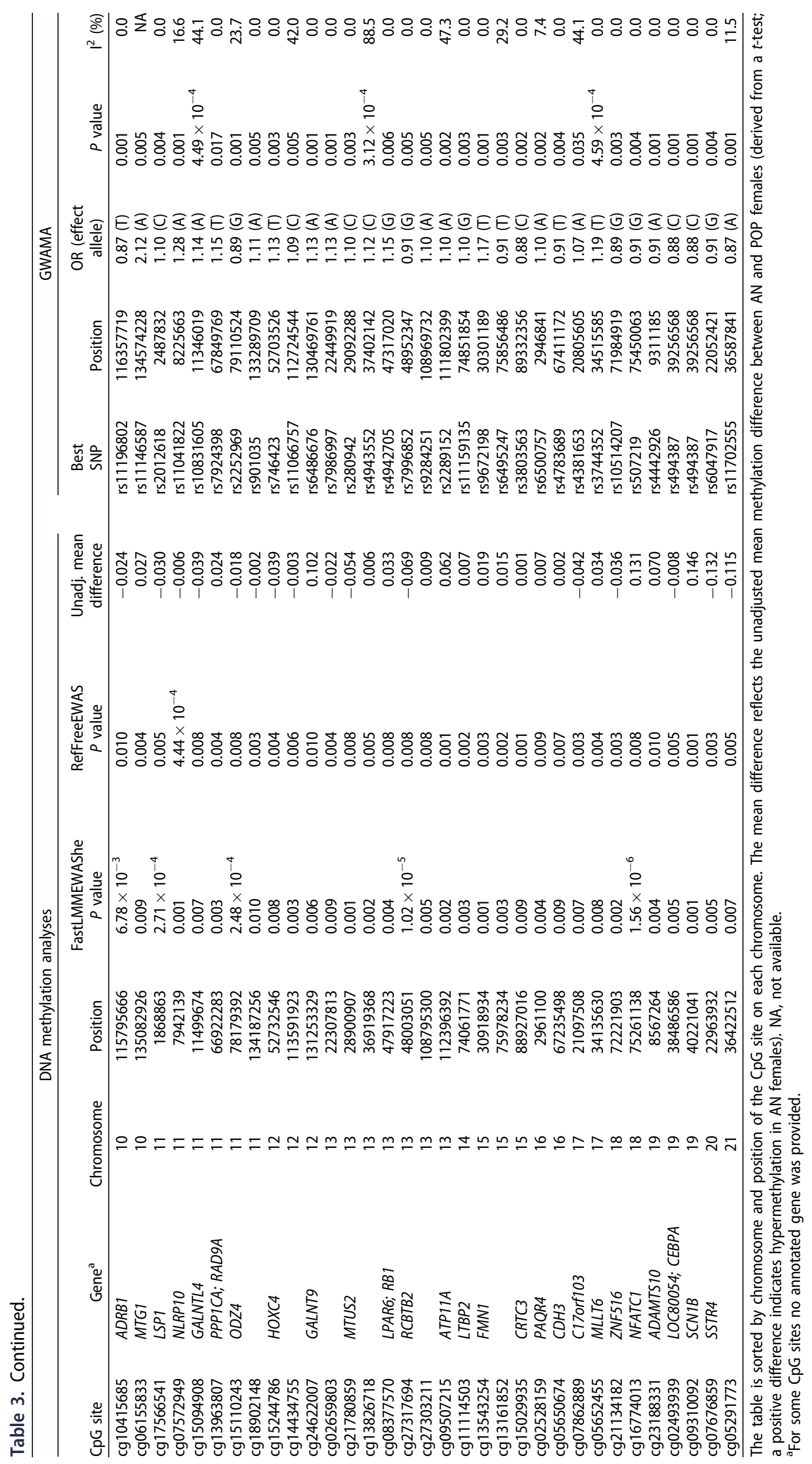


population-based controls. However, as we corrected for cell type distribution, the association signal is likely not due to cell type distribution effects. The $\mathrm{NR} 1 \mathrm{H} 3$ protein is highly expressed in visceral organs (liver, kidney and intestine) (Zhao \& Dahlman-Wright 2010; Basse et al. 2015). Studies in knock-out mice suggest an important role in the regulation of cholesterol homeostasis (Rippmann et al. 2009). Hence, this gene might well be relevant for starvation. Further epigenetic and functional analyses are warranted.

The comparison of the methylation patterns to the GCAN/WTCCC3 GWAMA (Boraska et al. 2014) yielded one SNP within $1 \mathrm{MB}$ of a CpG site (rs923768) with suggestive evidence for an association $\left(P=1.31 \times 10^{-6}\right)$. The SNP is located in an intron of the CSGALNACT1 gene. In our methylation analysis, differentially methylated $\mathrm{CpG}$ sites were detected for the comparison AN vs. POP. Studies in knock-out mice revealed a role of the gene product in cartilage formation. Thus, CSGALNACT1 is relevant for: (1) normal cartilage development (Sakai et al. 2007; Watanabe et al. 2010), (2) normal endochondral ossification, an essential process during foetal development by which bone tissue is formed, and (3) aggrecan metabolism (Sato et al. 2011). As reduced bone strength is reported in former AN patients (e.g., Mueller et al. 2015), our result might implicate an epigenetic process induced by the semi-starvation that leads to a reduced bone mass.

Two studies in humans also depicted interesting findings with respect to CSGALNACT1: (1) a mutation screen in human CSGALNACT1 in 114 patients with neuropathies (Guillain-Barré syndrome, chronic inflammatory demyelinating polyneuropathy, hereditary motor and sensory neuropathy and unknown aetiology) and 196 controls with other neurological diseases identified two novel non-synonymous mutations, not leading to $\mathrm{N}$-acetylgalactosamineT-II activities in two patients with neuropathies (His234Arg; Met509Arg) (Saigoh et al. 2011). (2) Furthermore, a suggestive effect was reported for rs7816924 $\left(P=2.11 \times 10^{-7}\right)$, an intronic variant of CSGALNACT1, in a GWAS of major depressive disorder (Hunter et al. 2013). This finding is interesting as depression is a frequent comorbidity in AN (Abbate-Daga et al. 2015).

Strengths of our study include: (1) We performed a high-throughput methylation association analysis and did not focus on single candidate genes. (2) We combined our high-throughput methylation analysis with the results of a systematic literature search and the results of a AN GWAMA on genomic variation at our consensus CpG sites. (3) We assessed the largest GWAMA for AN susceptibility assuming that either marker genotypes might influence epigenetic factors (Schalkwyk et al. 2010; Yuen \& Robinson 2011) or that DNA methylation might modify genetic effects resulting in heterogeneity when analysing the genetic effects in different populations (Abdolmaleky et al. 2004). (4) We followed up-to-date guidance to preprocess the raw data and accounted for cell type composition effects using two recently proposed reference-free methods. (5) The ignorance of the wellknown cell type composition effects (Fukudo et al. 1993; Saito et al. 1999; Castro et al. 2004; Sabel et al. 2013; Bühren et al. 2014) resulting from semi-starvation is not an option as presented by our analysis using CpG sites to map cell type differences between groups. In contrast, our analyses underline the importance of an additional modelling level of the cell type differences (presumably of general importance for highthroughput DNA methylation analyses).

However, limitations should also be noted: (1) We did not address particular sub-phenotypes or more quantitative endophenotype comparisons, as we had insufficient statistical power for these analyses. (2) Unfortunately, there was only a small overlap of consensus CpG sites detected by both analysis methods, which nevertheless clearly exceeded the overlap expected by chance. This missing overlap might either be due to our datasets (e.g., residual confounding, sample size) or due to differences between the methods (e.g., considered CpG sites, cell type effect modelling) as underlined by the inflation of small $P$ values for RefFreeEWAS. (3) We used a fairly liberal nominal significance level of 0.01 to screen for potentially differentially methylated CpG sites. Acknowledging that more stringent cut-offs would also lead to more falsenegative findings, we focused on consensus CpG sites detected by both reference-free methods, to reduce false-positive findings. (4) The POP were considerably older than both the AN patients and the lean females. As a consequence, some of the potentially differentially methylated $\mathrm{CpG}$ sites between AN and POP might be confounded by age. We cannot refute this argument even though investigation results indicated that the majority of DNA methylation marks seem to be relatively stable after birth (Bocklandt et al. 2011; Gordon et al. 2012; Johansson et al. 2013) and even though both our sensitivity analyses and cross-checks of the literature did not reveal any major age-related CpG sites among the consensus sites. (5) Similarly, we cannot refute that other effects such as BMI effects, or effects of hormone levels (Lomniczi et al. 2015; Osborne et al. 2016) or smoking might have in part confounded findings. Again we refer to the literature and to our sensitivity analyses to attenuate such 
arguments. (6) Clearly and probably the most limiting factors are our relatively small sample sizes for the high-throughput DNA methylation analyses and the choice of whole blood for the analyses. Furthermore, the sample size of the validation sample was again very small and very likely underpowered to make any firm conclusions. However, epigenetic studies on disease-discordant $M Z$ twin pairs (completely matched for genetics, age, sex, cohort effects, maternal influences and common environment) are more powerful in detecting disease-associated epigenetic differences than unrelated cases and controls with different lifehistories.

In sum, we confirmed hypermethylated $\mathrm{CpG}$ sites for AN near a gene with potential impact for AN, namely $T N X B$. Further studies should focus on this specific genetic region. In addition, we identified some evidence for differently methylated $C p G$ sites with potential relevance to starvation.

\section{Acknowledgements}

Funding for GCAN/WTCCC3 WT088827/Z/09 entitled "A genome-wide association study of anorexia nervosa". This work was funded by the Wellcome Trust (WT098051). Our analyses were supported by the "Deutsche Forschungsgemeinschaft" (DFG; HI865/2-1), the BMBF (01GS0820), "Landesmittel NRW, Landesprogramm Geschlechtergerechte Hochschulen", Academy of Finland (265240) and EPITRAIN - FP7-PEOPLE2012-ITN (316758). M.K., A.S. and the CSCC were supported by the Federal Ministry of Education and Research (BMBF), Germany, FKZs $01 E 01002$ and 01EO1502. M.O. was supported by the Academy of Finland (251316) and the Sigrid Juselius Foundation.

\section{Genetic consortium for anorexia nervosa (GCAN)}

Vesna Boraska Perica, Christopher S. Franklin, James A.B. Floyd, Laura M. Thornton, Laura M. Huckins, Lorraine Southam, N. William Rayner, loanna Tachmazidou, Kelly L. Klump, Janet Treasure, Cathryn M. Lewis, Ulrike Schmidt, Federica Tozzi, Kirsty Kiezebrink, Johannes Hebebrand, Philip Gorwood, Roger A.H. Adan, Martien J.H. Kas, Angela Favaro, Paolo Santonastaso, Fernando Fernández-Aranda, Monica Gratacos, Filip Rybakowski, Monika Dmitrzak-Weglarz, Jaakko Kaprio, Anna Keski-Rahkonen, Anu Raevuori-Helkamaa, Eric F. Van Furth, Margarita C.T. Slof-Op't Landt, James I. Hudson, Ted Reichborn-Kjennerud, Gun Peggy S. Knudsen, Palmiero Monteleone, Allan S. Kaplan, Andreas Karwautz, Hakon Hakonarson, Wade H. Berrettini, Yiran Guo, Dong Li, Nicholas J. Schork, Gen Komaki, Tetsuya Ando, Hidetoshi Inoko, Tõnu Esko, Krista Fischer, Katrin Männik, Andres Metspalu, Jessica H. Baker, Roger D. Cone, Jennifer Dackor, Janiece E. DeSocio, Christopher E. Hilliard, Julie K. O'Toole, Jacques Pantel, Jin P. Szatkiewicz, Chrysecolla Taico, Stephanie Zerwas, Sara E. Trace, Oliver S.P. Davis, Sietske Helder, Katharina Bühren,
Roland Burghardt, Martina de Zwaan, Karin Egberts, Stefan Ehrlich, Beate Herpertz-Dahlmann, Wolfgang Herzog, Hartmut Imgart, André Scherag, Susann Scherag, Stephan Zipfel, Claudette Boni, Nicolas Ramoz, Audrey Versini, Marek K. Brandys, Unna N. Danner, Carolien de Kove, Judith Hendriks, Bobby P.C. Koeleman, Roel A. Ophoff, Eric Strengman, Annemarie A. van Elburg, Alice Bruson, Maurizio Clementi, Daniela Degortes, Monica Forzan, Elena Tenconi, Elisa Docampo, Geòrgia Escaramí, Susana Jiménez-Murcia, Jolanta Lissowska, Andrzej Rajewski, Neonila Szeszenia-Dabrowska, Agnieszka Slopien, Joanna Hauser, Leila Karhunen, Ingrid Meulenbelt, P. Eline Slagboom, Alfonso Tortorella, Mario Maj, George Dedoussis, Dimitris Dikeos, Fragiskos Gonidakis, Konstantinos Tziouvas, Artemis Tsitsika, Hana Papezova, Lenka Slachtova, Debora Martaskova, James L. Kennedy, Robert D. Levitan, Zeynep Yilmaz, Julia Huemer, Doris Koubek, Elisabeth Merl, Gudrun Wagner, Paul Lichtenstein, Gerome Breen, Sarah Cohen-Woods, Anne Farmer, Peter McGuffin, Sven Cichon, Ina Giegling, Stefan Herms, Dan Rujescu, Stefan Schreiber, H-Erich Wichmann, Christian Dina, Rob Sladek, Giovanni Gambaro, Nicole Soranzo, Antonio Julia, Sara Marsal, Raquel Rabionet, Valerie Gaborieau, Danielle M. Dick, Aarno Palotie, Samuli Ripatti, Elisabeth Widén, Ole A. Andreassen, Thomas Espeseth, Astri Lundervold, Ivar Reinvang, Vidar M. Steen, Stephanie Le Hellard, Morten Mattingsdal, loanna Ntalla, Vladimir Bencko, Lenka Foretova, Vladimir Janout, Marie Navratilova, Steven Gallinger, Dalila Pinto, Stephen W. Scherer, Harald Aschauer, Laura Carlberg, Alexandra Schosser, Lars Alfredsson, Bo Ding, Lars Klareskog, Leonid Padyukov, Chris Finan, Gursharan Kalsi, Marion Roberts, Darren W. Logan, Leena Peltonen, Graham R.S. Ritchie, Jeff C. Barrett, Xavier Estivill, Anke Hinney, Patrick F. Sullivan, David A. Collier, Eleftheria Zeggini and Cynthia M. Bulik

\section{Wellcome trust case control consortium 3 (WTCCC3)}

Carl A. Anderson, Jeffrey C. Barrett, James A.B. Floyd, Christopher S. Franklin, Ralph McGinnis, Nicole Soranzo, Eleftheria Zeggini, Jennifer Sambrook, Jonathan Stephens, Willem H. Ouwehand, Wendy L. McArdle, Susan M. Ring, David P. Strachan, Graeme Alexander, Cynthia M. Bulik, David A. Collier, Peter J. Conlon, Anna Dominiczak, Audrey Duncanson, Adrian Hill, Cordelia Langford, Graham Lord, Alexander P. Maxwell, Linda Morgan, Leena Peltonen, Richard N. Sandford, Neil Sheerin, Frederik O. Vannberg, Hannah Blackburn, WeiMin Chen, Sarah Edkins, Mathew Gillman, Emma Gray, Sarah E. Hunt, Suna Nengut-Gumuscu, Simon Potter, Stephen S. Rich, Douglas Simpkin and Pamela Whittaker

\section{Statement of interest}

Dr. Bulik is a grant recipient from Shire. All other authors reported no biomedical financial interests or potential conflicts of interests.

\section{References}

Abbate-Daga G, Buzzichelli S, Marzola E, Aloi M, Amianto F, Fassino S. 2015. Does depression matter in 
neuropsychological performances in anorexia nervosa? A descriptive review. Int J Eat Disord. 48:736-745.

Abdolmaleky HM, Faraone SV, Glatt SJ, Tsuang MT. 2004. Meta-analysis of association between the T102C polymorphism of the $5 \mathrm{HT} 2 \mathrm{a}$ receptor gene and schizophrenia. Schizophr Res. 67:53-62.

Basse AL, Dixen K, Yadav R, Tygesen MP, Qvortrup K, Kristiansen K, Quistorff B, Gupta R, Wang J, Hansen JB. 2015. Global gene expression profiling of brown to white adipose tissue transformation in sheep reveals novel transcriptional components linked to adipose remodeling. BMC Genomics. 16:215.

Bocklandt S, Lin W, Sehl ME, Sanchez FJ, Sinsheimer JS, Horvath S, Vilain E. 2011. Epigenetic Predictor of Age. PloS One. 6e14821.

Booij L, Casey KF, Antunes JM, Szyf M, Joober R, Israël M, Steiger H. 2015. DNA methylation in individuals with anorexia nervosa and in matched normal-eater controls: $A$ genome-wide study. Int J Eat Disord. 48:874-882.

Boraska V, Franklin CS, Floyd JA, Thornton LM, Huckins LM, Southam L, Rayner NW, Tachmazidou I, Klump KL, Treasure $J$, et al. 2014. A genome-wide association study of anorexia nervosa. Mol Psychiatry. 19:1085-1094.

Bristow J, Tee MK, Gitelman SE, Mellon SH, Miller WL. 1993. Tenascin-X - a Novel Extracellular-Matrix Protein Encoded by the Human Xb Gene Overlapping P450c21b. J Cell Biol. 122:265-278.

Bühren K, Gartner L, Kennes LN, Seitz J, Hagenah U, HerpertzDahlmann B. 2014. Hematological changes in adolescent anorexia nervosa. Z Kinder Jugendpsychiatr Psychother. 42:19-26.

Campbell IC, Mill J, Uher R, Schmidt U. 2011. Eating disorders, gene-environment interactions and epigenetics. Neurosci Biobehav Rev. 35:784-793.

Castro J, Deulofeu R, Gila A, Puig J, Toro J. 2004. Persistence of nutritional deficiencies after short-term weight recovery in adolescents with anorexia nervosa. Int J Eat Disord. 35:169-178.

Chen WY, Kim MS, Shanbhag S, Arai A, VanRyzin C, McDonnell NB, Merke DP. 2009. The Phenotypic Spectrum of Contiguous Deletion of CYP21A2 and Tenascin XB: Quadricuspid Aortic Valve and Other Midline Defects. Am J Med Genet A. 149A:2803-2808.

Cipriani V, Leung HT, Plagno V, Bunce C, Khan JC, Shahid H, Moore AT, Harding SP, Bishop PN, Hayward C, et al. 2012. Genome-wide association study of age-related macular degeneration identifies associated variants in the TNXBFKBPL-NOTCH4 region of chromosome 6p21.3. Hum Mol Genet. 21:4138-4150.

Dedeurwaerder S, Defrance M, Calonne E, Denis H, Sotiriou C, Fuks F. 2011. Evaluation of the Infinium Methylation 450K technology. Epigenomics. 3:771-784.

Dick KJ, Nelson CP, Tsaprouni L, Sandling JK, Aissi D, Wahl S, Meduri E, Morange PE, Gagnon F, Grallert H, et al. 2014. DNA methylation and body-mass index: a genome-wide analysis. Lancet. 383:1990-1998.

Fraga MF, Ballestar E, Paz MF, Ropero S, Setien F, Ballestart ML, Heine-Suner D, Cigudosa JC, Urioste M, Benitez J, et al. 2005. Epigenetic differences arise during the lifetime of monozygotic twins. Proc Natl Acad Sci USA. 102:1060410609.
Fukudo S, Tanaka A, Muranaka M, Sasaki M, Iwahashi S, Nomura T, Tashiro A, Hoshino A. 1993. Reversal of Severe Leukopenia by Granulocyte Colony-Stimulating Factor in Anorexia Nervosa. Am J Med Sci. 305:314-317.

Gbadegesin RA, Brophy PD, Adeyemo A, Hall G, Gupta IR, Hains D, Bartkowiak B, Rabinovich CE, Chandrasekharappa S, Homstad A, et al. 2013. TNXB Mutations Can Cause Vesicoureteral Reflux. J Am Soc Nephrol. 24:1313-1322.

Gordon L, Joo JE, Powel JE, Ollikainen M, Novakovic B, Li X, Andronikos R, Cruickshank MN, Conneely KN, Smith AK, et al. 2012. Neonatal DNA methylation profile in human twins is specified by a complex interplay between intrauterine environmental and genetic factors, subject to tissue-specific influence. Genome Res. 22:1395-1406.

Haggarty P. 2015. Genetic and metabolic determinants of human epigenetic variation. Curr Opin Clin Nutr Metab Care. 18:334-338.

Heijmans BT, Tobi EW, Stein AD, Putter H, Blauw GJ, Susser ES, Slagboom PE, Lumey LH. 2008. Persistent epigenetic differences associated with prenatal exposure to famine in humans. Proc Natl Acad Sci USA. 105:17046-17049.

Hinney A, Barth N, Ziegler A, von Prittwitz S, Hamann A, Hennighausen K, Pirke KM, Heils A, Rosenkranz K, Roth $H$, et al. 1997. Serotonin transporter gene-linked polymorphic region: allele distributions in relationship to body weight and in anorexia nervosa. Life Sci. 61:295-303.

Houseman EA, Accomando WP, Koestler DC, Christensen BC, Marsit CJ, Nelson HH, Wiencke JK, Kelsey KT. 2012. DNA methylation arrays as surrogate measures of cell mixture distribution. BMC Bioinformatics. 13:86.

Houseman EA, Molitor J, Marsit CJ. 2014. Reference-free cell mixture adjustments in analysis of DNA methylation data. Bioinformatics. 301431-1439.

Hunter AM, Leuchter AF, Power RA, Muthen B, McGrath PJ, Lewis CM, Cook IA, Garriock HA, McGuffin P, Uher R, et al. 2013. A genome-wide association study of a sustained pattern of antidepressant response. J Psychiatr Res. 47:11571165.

Johansson A, Enroth S, Gyllensten U. 2013. Continuous aging of the human DNA methylome throughout the human lifespan. PLoS One. 8:e67378.

Kaprio J. 2013. The Finnish twin cohort study: an update. Twin Res Hum Genet. 16:157-162.

Keating ST, El-Osta A. 2015. Epigenetics and metabolism. Circul Res. 116:715-736.

Lomniczi A, Wright H, Ojeda SR. 2015. Epigenetic regulation of female puberty. Front Neuroendocrinol. 36:90-107.

Mueller SM, Immoos M, Anliker E, Drobnjak S, Boutellier U, Toigo M. 2015. Reduced bone strength and muscle force in women 27 years after anorexia nervosa. J Clin Endocrinol Metab. 100:2927-2933.

Osborne L, Clive M, Kimmel M, Gispen F, Guintivano J, Brown T, Cox O, Judy J, Meilman S, Braier A, et al. 2016. Replication of epigenetic postpartum depression biomarkers and variation with hormone levels. Neuropsychopharmacology. 41:1648-1658.

Pjetri E, Schmidt U, Kas MJ, Campbell IC. 2012. Epigenetics and eating disorders. Curr Opin Clin Nutr Metab Care. 15:330-335.

Rébé $C$, Filomenko $R$, Raveneau $M$, Chevriaux $A$, Ishibashi $M$, Lagrost L, Junien JL, Gambert P, Masson D. 2012. Identification of biological markers of liver $\mathrm{X}$ receptor (LXR) 
activation at the cell surface of human monocytes. PLoS One. 7:e48738.

Reinius LE, Acevedo N, Joerink M, Pershagen G, Dahlén S-E, Greco D, Söderhäll C, Scheynius A, Kere J. 2012. Differential DNA methylation in purified human blood cells: implications for cell lineage and studies on disease susceptibility. PLoS One. 7:e41361.

Rippmann JF, Schoelch C, Nolte T, Pavliska H, van Marle A, van Es $H$, Prestle J. 2009. Improved lipid profile through liver-specific knockdown of liver $\mathrm{X}$ receptor alpha in KKAy diabetic mice. J Lipid Res. 50:22-31.

Sabel AL, Gaudiani JL, Statland B, Mehler PS. 2013. Hematological abnormalities in severe anorexia nervosa. Ann Hematol. 92:605-613.

Saffrey R, Novakovic B, Wade TD. 2014. Assessing global and gene specific DNA methylation in anorexia nervosa: a pilot study. Int J Eat Disord. 47:206-210.

Saigoh K, Izumikawa T, Koike T, Shimizu J, Kitagawa H, Kusunoki S. 2011. Chondroitin beta-1,4-N-acetylgalactosaminyltransferase- 1 missense mutations are associated with neuropathies. J Hum Genet. 56:143-146.

Saito S, Kita K-i, Morioka CY, Watanabe A. 1999. Rapid recovery from anorexia nervosa after a life-threatening episode with severe thrombocytopenia: Report of three cases. Int J Eat Disord. 25:113-118.

Sakai K, Kimata K, Sato T, Gotoh M, Narimatsu H, Shinomiya K, Watanabe H. 2007. Chondroitin sulfate $\mathrm{N}$-acetylgalactosaminyltransferase- 1 plays a critical role in chondroitin sulfate synthesis in cartilage. J Biol Chem. 282:4152-4161.

Sato T, Kudo T, Ikehara Y, Ogawa H, Hirano T, Kiyohara K, Hagiwara K, Togayachi A, Ema M, Takahashi S, et al. 2011. Chondroitin sulfate $\mathrm{N}$-acetylgalactosaminyltransferase 1 is necessary for normal endochondral ossification and aggrecan metabolism. J Biol Chem. 286:5803-5812.

Schalkwyk LC, Meaburn EL, Smith R, Dempster EL, Jeffries AR, Davies MN, Plomin R, Mill J. 2010. Allelic skewing of DNA methylation is widespread across the genome. Am J Hum Genet. 86:196-212.

Touleimat N, Tost J. 2012. Complete pipeline for Infinium (R) Human Methylation 450K BeadChip data processing using subset quantile normalization for accurate DNA methylation estimation. Epigenomics. 4:325-341.

Watanabe Y, Takeuchi K, Onaga SH, Sato M, Tsujita M, Abe M, Natsume R, Li MQ, Furuichi T, Saeki M, et al. 2010. Chondroitin sulfate $\mathrm{N}$-acetylgalactosaminyltransferase- 1 is required for normal cartilage development. Biochem J. 432:47-55.

Weissensteiner T, Lanchbury JS. 1997. Allelic polymorphism of two multifunctional regions in the central human MHC: Tenascin $X, X B-S$ and $Y B$, and their duplicated fragments $X A$ and YA. Eur J Immunogenet. 24:201-209.

Yuan Y, Nymoen DA, Stavnes HT, Rosnes AK, Bjorang O, Wu CY, Nesland JM, Davidson B. 2009. Tenascin-X is a novel diagnostic marker of malignant mesothelioma. Am J Surg Pathol. 33:1673-1682.

Yuen RKC, Robinson WP. 2011. Review: a high capacity of the human placenta for genetic and epigenetic variation: Implications for assessing pregnancy outcome. Placenta. 32:S136-S141.

Zhao CY, Dahlman-Wright K. 2010. Liver X receptor in cholesterol metabolism. J Endocrinol. 204:233-240.

Zou J, Lippert C, Heckerman D, Aryee M, Listgarten J. 2014. Epigenome-wide association studies without the need for cell-type composition. Nat Methods. 11:309-311. 